auricular beat, but somewhat later there was complete dissociation between the two pairs of cavities. Before the patient left the hospital, the relation between the auricles and ventricles was normal. Electric curves were taken on December 19, I9I I, when the ventricles responded to every auricular contraction.

The curves which are of interest date from the period of complete auriculo-ventricular dissociation. Each ventricular complex is represented by $R$-, $S$-, and $T$-waves. They vary from each other in that, when the $R$ - is large, the $S$-wave is small; and that when the $S$-wave is large, the $R$-wave is small. The $R$-wave gradually increases in size and then gradually diminishes, when the $S$-wave gradually increases in size and as gradually diminishes. If the apexes of succeeding $R$ - and $S$-waves, $i$. e., the significant wave in each complex were joined, a waved line would result. So diagrammatic a sequence as this did not occur frequently, but there was a tendency to approximate to this description. Sometimes the transition from complexes of one type to those of another was abrupt. The time between the complexes was almost equal, except at points of transition, when it was reduced.

It is concluded from the variation in the shape of the complexes, that they are responses to stimuli arising at levels in the heart varying from some supraventricular position to the apex. The explanation of the shortened time at points of transition is difficult. No hypothesis yet suggested is satisfactory. The fact that there was merely a temporary and not a permanent dissociation may be a significant factor, although the $P-R$ interval associated with the transitions is not of uniform length. ( $P$ is the wave representing auricular systole.)

\title{
$8(627)$
}

\section{Further observations on the tolerance of gases by the circulatory apparatus.}

\section{By J. P. AtKInson and C. B. FitzPatrick.}

[From the Department of Health, City of New York.]

In the New York Medical Journal of November 26 and at the New Haven meeting of December, 1910, we gave observations on the quantities of gas (air) tolerated and the apparent relation of 
the adrenals to this tolerance. We have further determined that section of the cord between the fifth and sixth cervical vertebræ interferes with this tolerance of gas (air). After the removal of the adrenals from two dogs carbon dioxide was tolerated up to 942 c.c. in one case and 952 c.c. in the other, in I hour and 23 minutes.

Two dogs were injected with nitrogen. ${ }^{1}$ One received 32 c.c. in six minutes with marked depressions; the animal lived. Panting occurred. The other received 272 c.c. within 22 minutes and died; panting was not noticed. The lungs were collapsed at autopsy.

The tolerance of oxygen was tested on two dogs. One received I50 c.c. slowly and survived. The other received 312 c.c. in $231 / 2$ minutes and died. During the oxygen experiments, panting occurred. The lungs were practically normal.

The tolerance of hydrogen was tested in two dogs. In one 184 c.c. in 8 c.c. volumes were injected in $13 \frac{1}{2}$ minutes. Each injection caused a moderate depression. 60 c.c. were given in 20 c.c. volumes in $31 / 2$ minutes, and 20 c.c. in 5 c.c. volumes. The large volumes caused great depression and almost death. In the other dog 90 c.c. were injected in 7 minutes in 8 c.c. volumes without harmful results.

Sulphuretted hydrogen was used to study elimination on two dogs. Three c.c. saturated $\mathrm{H}_{2} \mathrm{~S}$ water were injected into the femoral vein. The breath almost immediately blackened lead acetate. Twenty-five minutes after tying and clamping off the adrenals 3 c.c. more of saturated $\mathrm{H}_{2} \mathrm{~S}$ water was injected and a similar result was obtained. Ioo c.c. of $\mathrm{H}_{2} \mathrm{~S}$ water introduced into the rectum did not give the reaction at the mouth in 25 minutes. In the other dog 30 c.c. of $\mathrm{H}_{2} \mathrm{~S}$ water were injected into the duodenum. In 8 minutes it was detected at the mouth.

1 In every case morphin sulphate was given before etherization of the dog. Each dog was killed and autopsied after the experiment. 